\title{
Uma proposta de metodologia para adaptação de OA usando critérios de acessibilidade
}

\section{Cristiani de Oliveira Dias - Programa de Pós-Graduação em Educação - cristianideoliveiradias@gmail.com}

\section{Liliana Maria Passerino - Programa de Pós-Graduação em Educação - lilianap@cinted.ufrgs.br}

\begin{abstract}
The intent of this paper is to study the learning objects from another perspective, be available in its content and format for all the people / citizens, having special needs or not. Therefore, it is planned to build a methodology for developing object oriented software following the fusion method that facilitates the development of learning objects. Throughout the text we present the development steps of accessible LO as well the final considerations of the work.
\end{abstract}

Resumo. O objetivo do presente artigo é discutir o desenvolvimento de objetos de aprendizagem sob outra perspectiva, a da acessibilidade, permitindo que seu conteúdo e formato seja acessível para todas as pessoas com necessidades especiais ou não. Parte-se então da construção de uma metodologia de desenvolvimento de software orientado a objetos seguindo o método fusion que facilita o desenvolvimento de objetos de aprendizagem. No decorrer do texto apresentamos as etapas de desenvolvimento do OA acessível assim como as considerações finais do trabalho.

\section{Introdução}

O autor Zabala (1998) apresenta um estudo sobre as diversas variáveis que configuram a prática educativa, as principais são planejamento, avaliação, intervenção, relações interativas entre aluno/professor, recursos didáticos, conteúdos de aprendizagem, organização social e temporal do espaço educativo tudo isso em torno de determinadas intenções educacionais. Mas, apesar das diferentes variáveis que configuram a prática educativa é possível investigar a mesma, escolhendo uma unidade de análise que represente a complexidade desse processo, propondo para tal a atividade como uma unidade básica. Dentro dessa unidade, conteúdos e recursos didáticos têm função de destaque enquanto signos e instrumentos de mediação, pois para Vygotsky (1988 p.43) o sujeito não é apenas ativo, mas interativo, construindo conhecimentos e se constituindo a partir de relações intra e interpessoais mediadas por instrumentos e signos.

Em particular, os recursos didáticos utilizados no processo educativo funcionam como "configuradores" da informação que poderá ser transformada em conhecimento, após um dinâmico e longo processo de transformações, apropriações e internalizações que os alunos farão com apoio (mediação) do professor. Esses "configuradores", longe de serem recursos neutros são essenciais para o acesso e a compreensão da informação e, portanto a "porta de entrada" ao conhecimento. Diferentes tipos de materiais educacionais, como, livro texto, vídeos, mapas, entre outros, formam o que denominamos de suportes midiáticos. O suporte midiático mais popular na educação é o livro texto. Já é uma tradição o livro texto na sala de aula, porém essa hegemonia 
encontra-se atualmente ameaçada por outros suportes midiáticos do tipo digital, o mais poderoso deles a Web $2.0 \mathrm{com}$ materiais interativos e hipertextuais que tem se consumado na educação em todos os níveis ${ }^{1 .}$

Essa proliferação de material educacional no formato digital nos últimos anos como blogs ou Wikipédia em iniciativas individuais ou coletivas com as quais o professor passa a ser autor e produtor juntamente com seus alunos leva a uma preocupação com relação à qualidade de seus conteúdos. Esta preocupação levou o Governo Federal a criar políticas de fomento e divulgação de Objetos de Aprendizagem financiados com verbas públicas. Entre essas políticas pode-se mencionar o RIVED ${ }^{2} \mathrm{e}$ após expansão do projeto surge o Fábrica Virtual ${ }^{3 .}$

A distribuição desses materiais educacionais produzidos por Instituições de Ensino Superior atendem a grande número de escolas públicas, já que são distribuídas em formatos eletrônicos via Secretaria da Educação de cada Estado.

Porém, a qualidade em termos de conteúdo não é a única variável que deveria ser considerada nestas políticas públicas. Considerando que esse conteúdo e seu suporte constituem a "porta de entrada" ao processo educativo, precisamos nos preocupar com o acesso e compreensão da informação neles contida de forma a atender à diversidade da sala de aula. Nesse sentido, pesquisas ${ }^{4}$ feitas sobre objetos de aprendizagem disponíveis para uso na sala de aula nos portais como RIVED mostrou que praticamente a totalidade desses objetos não contempla recomendações mínimas de acessibilidade [Passerino, Behar e Dias, 2009]. Diante desta situação, foi iniciada uma pesquisa com a finalidade de pensar a adaptação de Objetos de Aprendizagem para atender os critérios de acessibilidade e propor uma metodologia para construção de OAs acessíveis. O presente artigo apresenta a metodologia proposta suas implicações em termos de engenharia de software e os resultados educacionais esperados destacando que a proposta metodológica sugerida poderá também servir para nortear desenvolvimentos computacionais para outras áreas buscando respeitar a diversidade de forma e garantir a acessibilidade para todos.

\section{Critérios de Acessibilidade}

Falar de acessibilidade significa construir uma sociedade de plena participação e igualdade e ter em seus princípios a interação efetiva de todos os cidadãos. Nessa perspectiva, é fundamental a construção de políticas de inclusão para o reconhecimento

1 Dados da Comissão Européia sobre uso das TIC nas escolas em 2006 mostra que 98,7 \% dos professores utilizam computador para suas atividades profissionais, sendo que $74,5 \%$ o fazem nas suas aulas e o restante para planejamento e estudos. (European Comission, 2006)

Projeto que utiliza a produção de recursos pedagógicos digitais como meio de ajudar o desenvolvimento do raciocínio e o pensamento crítico dos estudantes é o RIVED Rede Interativa Virtual de Educação, associando o potencial da informática às novas abordagens pedagógicas.

3 Que tem como propósito intensificar e transferir o processo de desenvolvimento e produção de recursos educacionais digitais (na forma de objetos de aprendizagem) da SEED (Secretaria de Educação a Distância) para as Instituições de Ensino Superior (RIVED, 2008).

$4 \quad$ Pesquisa feita para Projeto OBAA - Objetos de Aprendizagem Interoperáveis apoiado pelo FINEP/MCT. Publicação de artigo em evento internacional.

V. $7 \mathrm{~N}^{\mathrm{o}}$ 3, dezembro, 2009 
da diferença e exista uma sociedade em que todos devem participar, com direito de igualdade e de acordo com suas especificidades.

Segundo Dias (2003) a acessibilidade em materiais digitais significa que qualquer pessoa, usando qualquer tipo de tecnologia de navegação (gráficos, textuais, especiais para cegos ou para sistemas de computação móvel) deve ser capaz de visitar interagir com qualquer conteúdo, compreendendo inteiramente as informações neles apresentadas.

Portanto, acessibilidade não significa transformar um material educacional interativo e gráfico (para atender alunos videntes) em um material educacional textual (para alunos com deficiência visual). Será que realmente quero incluir os meus alunos com esse tipo de material? Com certeza não. Pois acessibilidade significa transformar em acessível qualquer produto, sem perder suas propriedades e conteúdos.

Alguns critérios são importantes na construção de objetos de aprendizagem ou qualquer material educacional. Nessa tabela 1 ficam descritas algumas informações básicas a partir de recomendações da WCAG de 2008 de conteúdos, foram feitas adaptações para contemplar e auxiliar a construção de objetos de aprendizagem.

Tabela 1 Fonte: W3C, 2008 (http://www.w3.org/WAI/intro/components.php)

$$
\text { Princípio 1: Perceptivel }
$$

A informação e os componentes da interface do usuário têm de ser apresentados aos usuários em formas que eles possam perceber

\begin{tabular}{|c|c|c|c|}
\hline $\begin{array}{c}\text { Alternativas } \\
\text { em Texto }\end{array}$ & $\begin{array}{c}\text { Mídias com base no } \\
\text { tempo }\end{array}$ & Adaptável & Discernível \\
\hline $\begin{array}{l}\text { Isso significa } \\
\text { que se o } \\
\text { material } \\
\text { educacional } \\
\text { que está sendo } \\
\text { construído, } \\
\text { tenha muitas } \\
\text { imagens ou } \\
\text { animações que } \\
\text { sejam } \\
\text { fornecidas } \\
\text { alternativas em } \\
\text { texto para } \\
\text { qualquer } \\
\text { conteúdo não } \\
\text { textual. }\end{array}$ & $\begin{array}{l}\text { Fornecer } \\
\text { alternativas para } \\
\text { mídias com base no } \\
\text { tempo. Isso } \\
\text { significa que sejam } \\
\text { disponibilizadas } \\
\text { mídias alternativas } \\
\text { dentro conteúdo } \\
\text { apresentado. Que } \\
\text { possa ser um vídeo, } \\
\text { áudio e que } \\
\text { apresente também } \\
\text { legendas no caso de } \\
\text { vídeo e } \\
\text { autodescrição no } \\
\text { caso das duas } \\
\text { mídias. }\end{array}$ & $\begin{array}{l}\text { Criar } \\
\text { conteúdos que } \\
\text { possam ser } \\
\text { apresentados } \\
\text { de diferentes } \\
\text { maneiras (por } \\
\text { ex., um layout } \\
\text { mais simples) } \\
\text { sem perder } \\
\text { informação ou } \\
\text { estrutura. }\end{array}$ & $\begin{array}{l}\text { Facilitar a } \\
\text { audição e a } \\
\text { visualização de } \\
\text { conteúdos aos } \\
\text { usuários, } \\
\text { incluindo a } \\
\text { separação do } \\
\text { primeiro plano } \\
\text { e do plano de } \\
\text { fundo. }\end{array}$ \\
\hline \multicolumn{4}{|c|}{ Princípio 2: Operável } \\
\hline $\begin{array}{c}\text { Acessível por } \\
\text { Teclado }\end{array}$ & \multicolumn{2}{|c|}{ Tempo Suficiente } & avegável \\
\hline
\end{tabular}




\begin{tabular}{|c|c|c|c|c|}
\hline \multicolumn{2}{|c|}{$\begin{array}{l}\text { Fazer com que } \\
\text { toda a } \\
\text { funcionalidade } \\
\text { fique disponível a } \\
\text { partir do teclado. }\end{array}$} & \multicolumn{2}{|c|}{$\begin{array}{l}\text { Fornecer tempo } \\
\text { suficiente aos } \\
\text { usuários para lerem e } \\
\text { utilizarem o } \\
\text { conteúdo. Que o } \\
\text { aluno possa "ajustar" } \\
\text { o tempo de execução } \\
\text { das atividades. }\end{array}$} & $\begin{array}{l}\text { Fornecer formas de ajudar } \\
\text { os usuários a navegar, } \\
\text { localizar conteúdos e } \\
\text { determinar o local onde } \\
\text { estão. }\end{array}$ \\
\hline \multicolumn{3}{|c|}{$\begin{array}{l}\text { Princípio 3: Compreensível } \\
\text { A informação e a operação da } \\
\text { interface de usuário têm de ser } \\
\text { compreensíveis }\end{array}$} & \multirow{2}{*}{\multicolumn{2}{|c|}{$\begin{array}{c}\text { Princípio 4: Robusto } \\
\text { O conteúdo tem de ser robusto o } \\
\text { suficiente para poder ser } \\
\text { interpretado de forma concisa } \\
\text { por diversos agentes do usuário, } \\
\text { incluindo tecnologias assistivas. }\end{array}$}} \\
\hline Legível & & Previsível & & \\
\hline $\begin{array}{l}\text { Tornar o } \\
\text { conteúdo de } \\
\text { texto legível e } \\
\text { compreensível. } \\
\text { Prestar atenção } \\
\text { para não } \\
\text { utilizar termos } \\
\text { e linguagens } \\
\text { que atendam } \\
\text { somente a um } \\
\text { público } \\
\text { específico. }\end{array}$ & $\begin{array}{l}\text { Que } \\
\text { pág } \\
\text { um } \\
\text { nav } \\
\text { con } \\
\text { repe } \\
\text { repi } \\
\text { de } r \\
\text { bro }\end{array}$ & $\begin{array}{l}\text { seu material ou } \\
\text { ina web tenha } \\
\text { sistema de } \\
\text { egação } \\
\text { sistente e não } \\
\text { etido (que } \\
\text { itam os sistemas } \\
\text { navegação do } \\
\text { wser) }\end{array}$ & & $\begin{array}{l}\text { aximizar a compatibilidade } \\
\text { a atuais e futuros agentes de } \\
\text { lário, incluindo tecnologias } \\
\text { istivas. Prestar atenção para } \\
\text { r páginas de marcação como } \\
\text { HTML e que as mesmas } \\
\text { esentem as marcas de início } \\
\text { de fim completas e que os } \\
\text { nentos não contêm atributos } \\
\text { duplicados. }\end{array}$ \\
\hline
\end{tabular}

Nesse paradigma de educação inclusiva, os recursos de tecnologia de informação e comunicação (TIC) utilizados no processo de ensino e aprendizagem precisam também ser acessíveis. Incluem-se nesse caso os objetos de aprendizagem (OAs), definidos por Willey (2002) como pequenos componentes digitais reutilizáveis para uso na educação.

O conceito de reusabilidade se deu a partir da utilização da programação orientada a objetos, onde todo o código ou parte dele pode ser utilizado no desenvolvimento ou adaptação de outro programa. Isso permite que dependendo do público-alvo que utilizará o objeto, ele pode ser remodelado atendendo essa necessidade [Takahashi,1990 p.57].

Foram feitos pesquisas sobre objetos de aprendizagem acessíveis e tivemos um resultado pequeno e com algumas críticas a respeito. Para nós, objetos de aprendizagem que atendem à diversidade significam que sejam construídos desde seu projeto pensando em atender a Pessoas com Necessidades Educativas Especiais (PNEEs). Essa preocupação não é recente, diversas pesquisas abordam a questão da acessibilidade e da importância de existir materiais adaptados às diferentes necessidades dos alunos [Poletto, 2007]. Porém, na maioria das vezes a proposta é re-adaptar o material para 
uma determinada necessidade ou limitação. Como por exemplo, os materiais educacionais desenvolvidos pelo Cefet-BG ${ }^{5}$.

Em suma, os materiais que se dizem acessíveis, são na verdade "novas" versões diferenciadas dos materiais educacionais originais e que de alguma forma exclui esse aluno com Necessidades Especiais, ao não permitir a interação no mesmo tipo de material, privando o aluno ao acesso à informação original em sua completude. Obviamente não se trata de manter a versão original do material de forma que não permita a interação desses alunos, mas pensar tal material de forma convergente midiaticamente falando.

\section{Adaptação de Objetos de Aprendizagem: primeira abordagem}

Para atender a esses objetivos, foi idealizada uma pesquisa qualitativa com foco em análise de normas para definições de requisitos e desenvolvimento tecnológico com pesquisa de campo para validação. A pesquisa contempla quatro etapas:

-Etapa 1: Escolha do Objeto de Aprendizagem: esta etapa se propõe mostrar que os critérios para páginas web podem e devem ser aplicados para o OAs e para isso foi desenvolvido um estudo de caso voltado para um objeto em particular. O objeto selecionado foi "Banca do Quincas" jogo premiado no Concurso RIVED 2007 e foi desenvolvido pela Universidade de Salvador (UNIFACS), cuja principal característica é de ser um OA aberto do tipo simulação no qual se administra uma banca que vende produtos $^{6}$. A presente pesquisa é decorrente de uma parceria entre grupos de pesquisas da Universidade Federal do Rio Grande do $\mathrm{Sul}^{7}$ e da Universidade de Salvador.

\section{-Etapa 2: Processos de reengenharia do objeto de aprendizagem}

De acordo com os autores Chilkofsky e Cross (1990) reengenharia pode ser conceituada como: a) engenharia direta: processo tradicional que parte de abstrações de alto nível e projetos lógicos e chega a implementação do sistema; b) engenharia reversa que cria representações de um sistema em uma outra forma, ou em um nível mais alto de abstração; c) reestruturação que representa a transformação de uma representação em outro no mesmo nível de abstração, preservando o comportamento externo do sistema e por fim. A reengenharia propriamente dita é composta pela engenharia reversa e também pela engenharia direta ou pela reestruturação. Em contraste com a reestruturação, a reengenharia pode envolver modificações de funcionalidade ou de técnicas de implementação. Coleman (1996, p.288) destaca que

"O processo de reengenharia pode ser definido em três cenários diferenciados: (1) mudança completa na técnica de implementação, sem alterar a funcionalidade do sistema; (2) situação onde ainda não há qualquer tipo de mudança na funcionalidade, sendo apenas requerida uma mudança parcial

\footnotetext{
5 Materiais criados e publicados no endereço:

http://www.bento.ifrs.edu.br/ept/oa/regradetres/\#iniciomenu

Para mais informações referentes a este OA sugere-se consultar Santanchè (2008). http://logames.sourceforge.net/

7 Do grupo de pesquisa e de projeto de mestrado do PPGedu e projeto aprovado pela SEAD/UFRGS Edital 112009.
}

V. $7 \mathrm{~N}^{\mathrm{o}}$ 3, dezembro, 2009 


\section{na técnica de implementação e; (3) situação onde também haja alterações na} funcionalidade”.

A intenção dessa reengenharia no OA escolhido foi a de criar funcionalidades novas, a partir das existentes modificando o código-fonte para adaptar acessivelmente esse OA. Prevê algumas descrições no processo de reengenharia e faremos a citação para confirmar a escolha do método de reengenharia. Seguindo as ideias de Coleman (1996) optamos pelo cenário 2 na qual foi realizada uma adição na implementação do sistema, sem afetar com isso a sua funcionalidade. No nosso caso, a implementação consistiu numa ponte ${ }^{8}$ de comunicação chamada Java Access Bridge, que permite a comunicação entre leitores de tela ${ }^{9}$ com o OA desenvolvido e que primeiramente foi testado com sujeitos cegos e com baixa visão para comprovar sua limitação. Isso facilitou a inclusão posterior da API de acessibilidade que permitirá incorporar outros recursos de acessibilidade ao OA (outras deficiências). Para a construção desse processo de implementação de novos recursos, foram feitos estudos de incorporação de novos requisitos, que significa que serão feitas modificações nos modelos de análise de acordo com os novos requisitos. Fazendo que sejam produzidos modelos de análise compostos por três subconjuntos representando, respectivamente: novos objetos, objetos antigos envolvidos com a nova funcionalidade e os objetos antigos [Coleman, 1996 p.290].

Além da API de acessibilidade, a Sun disponibiliza o Java Accessibility Utility, que são classes desenvolvidas para ajudar as tecnologias assistivas a acessar programas rodando na máquina virtual Java e a entender a API de acessibilidade (Sun 1999), sendo importante o profissional que irá desenvolver o Objeto em Java, que o desenvolva pensando nos requisitos de acessibilidade do programa assim como nas Tecnologias Assistivas a serem utilizadas com ele.

Como os objetos de aprendizagem deveriam ser construídos na perspectiva de construção de sistemas orientados a objetos, optamos pelo método fusion ${ }^{10}$ para desenvolvimento de software, pois os outros métodos estão associados a apenas partes do estado do sistema em um modelo orientado a objeto. O método fusion vem atingir esses problemas, fornecendo todos os recursos para análise, projeto e implementação. As notações do fusion permitem, de forma sistemática, descobrir e preservar as estruturas dos objetos do sistema [Coleman, 1996]. O método Fusion adota a divisão do processo de desenvolvimento em análise, projeto e implementação. Sendo o processo de

$8 \quad$ bridge de comunicação Java, ou seja, caso um desenvolvedor deseja integrar sua aplicação Java com o Gnome basta que ele faça uso do Java Access Bridge for Gnome - JABG. Para que a ponte possa permitir que as aplicações Java façam acesso ao framework de acessibilidade do Gnome, especificadamente a AT-SPI desta forma o Orca se torna apto a fazer leitura das aplicações Java.

Para atender a esses alunos existem os programas chamados Leitores de Tela que são sintetizadores de voz que farão a leitura dos conteúdos apresentados na tela do computador. Como exemplo desses programas, temos DOSVOX - inicialmente proposto como um sistema operacional para deficientes visuais pelo Núcleo de Computação Eletrônica da Universidade Federal do Rio de Janeiro, este programa tem hoje versão para DOS e Windows (WINVOX) e engloba editor de texto, módulo de impressão em BRAILLE, agenda, jogos e diversos aplicativos. Para muitos usuários, ele é considerado o programa básico para inserir o deficiente visual no uso da informática. O programa está disponível gratuitamente no endereço: http://intervox.nce.ufrj.br/dosvox/. (Melo, 2008)

10 Método Fusion é um método de desenvolvimento de software voltado para a produção de software orientado e a objetos. Trata-se de um método de completa abrangência, fornecendo todos os recursos para analise, projeto e implementação. As notações do Fusion permitem, de forma sistemática, descobrir e preservar as estruturas dos objetos do sistema. (Coleman, 1996)

V. $7 \mathrm{~N}^{\mathrm{o}}$ 3, dezembro, 2009 
captura de requisitos será feita pelo usuário, que deve fornecer o documento inicial de requisitos. Esse processo está dividido então em:

-Fase de análise: onde é definido o comportamento esperado do sistema.

Foram feitos estudos a partir do código-fonte do objeto e do diagrama UML criado pela equipe de desenvolvimento inicial.

-Fase de projeto: como o sistema será implementado pelo comportamento, em tempo de execução, dos objetos em interação [Coleman, 1996 p.287]. Na construção do objeto de aprendizagem essa fase foi feita pelo grupo desenvolvedor inicial. No objeto de aprendizagem, apesar dele ter código-fonte pronto e modelagem UML já pronta, foi feita uma modelagem DE-R para verificar quais as funcionalidades do objeto e os processos de interação do objeto com usuário. A figura 4 mostra essas funções.

O processo de reengenharia desse objeto prevê que essa tabela que faz toda a interação do objeto com o usuário se transforme em um formulário com a mesma funcionalidade, porém esse formulário passa a ser acessível aos leitores de tela, caso que não acontece com as tabelas.

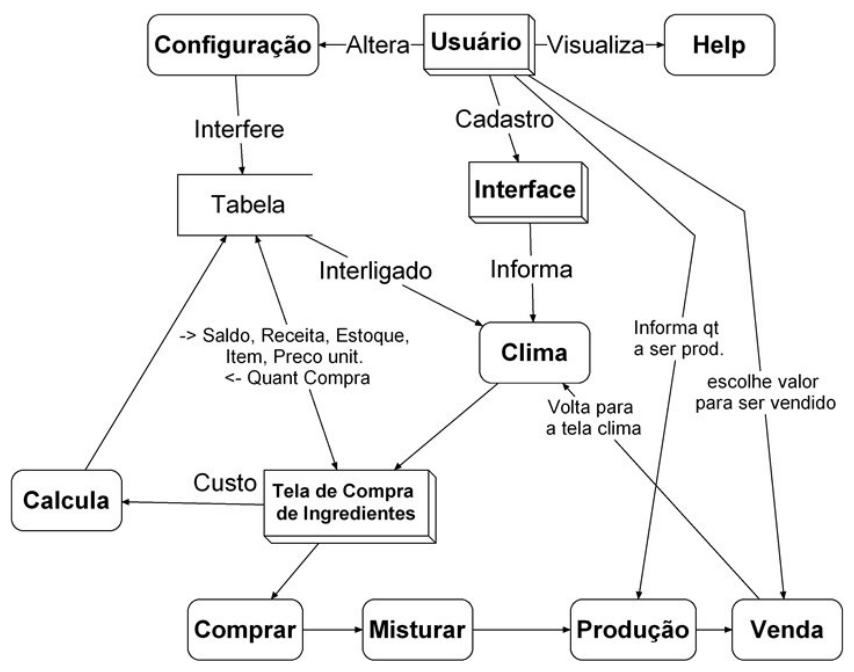

Figura 4 - Diagrama E-R do OA Objeto Banca do Quincas

-Fase de implementação: definida a implementação do código-fonte, com herança, atributos, referências, classes e interação do objeto [Coleman, 1996 p.287]. Essa fase encontra-se em processo de desenvolvimento, para que o código-fonte atenda aos requisitos de acessibilidade. Para isso, no nosso projeto, estamos implementando uma ponte de comunicação chamada Java Access Bridge, que permite a comunicação entre leitores de tela com o OA desenvolvido (primeiramente para ser testado com sujeitos cegos e com baixa visão). Isso facilitará a inclusão posterior da API de acessibilidade que permitirá incorporar outros recursos de acessibilidade ao OA (outras deficiências).

Todas as etapas de desenvolvimento podem ser sintetizadas num workflow conforme figura 5. Como podemos ver nesse workflow, os processos são conjuntos e o processo de validação direta (validação feita com sujeitos) está presente em todo o 
desenvolvimento. $\mathrm{O}$ processo de acessibilizar o software também fica presente no inicio e fim do desenvolvimento para potencializar o processo de desenvolvimento.

O desenvolvimento e readaptação podem ser utilizados para construção tanto de software como para objetos de aprendizagem.

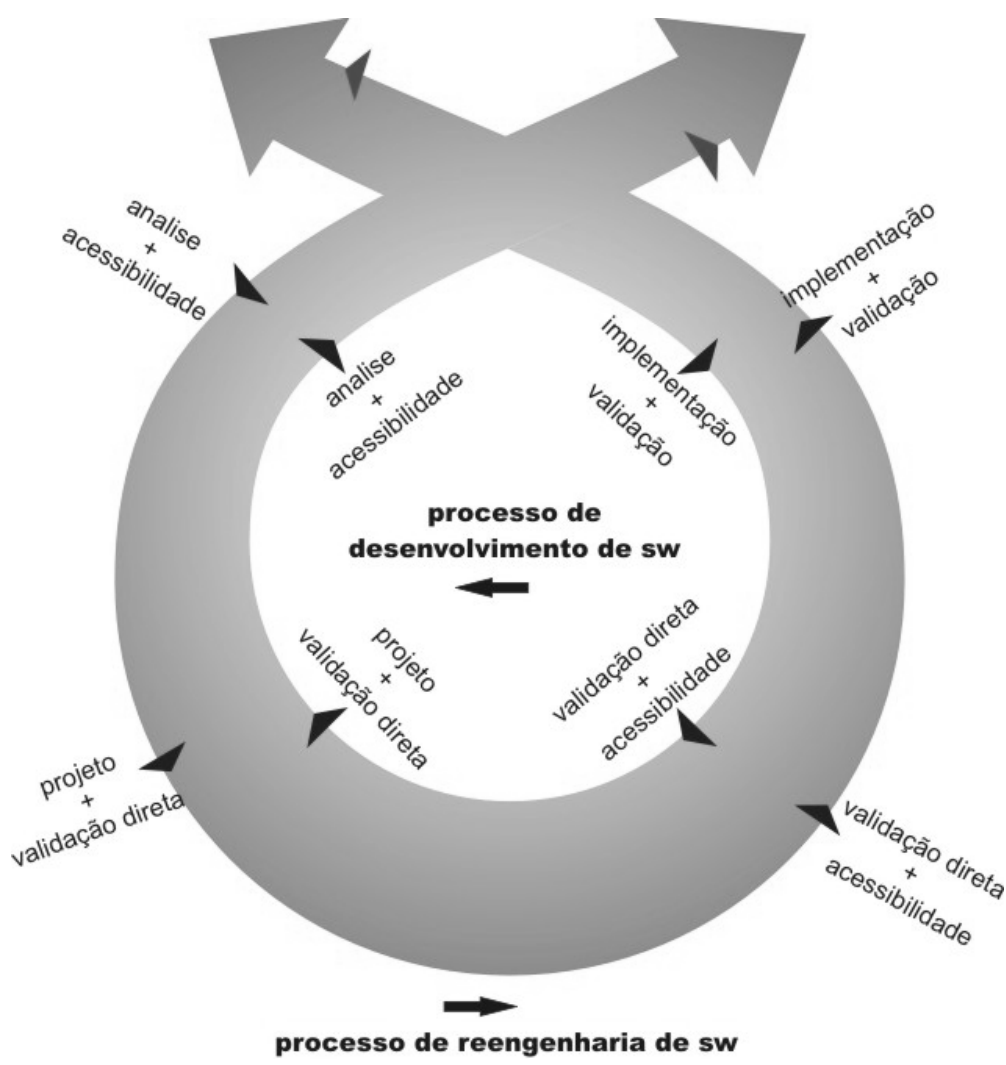

Figura 5: Workflow de desenvolvimento de Software

\section{- Etapa 3: Processo de avaliação do objeto de aprendizagem com usuários com baixa visão}

Para avaliar esse objeto de aprendizagem antes do processo de reestruturação do mesmo, foi testado com sujeito com baixa visão (visão periférica mais precisamente) onde foi avaliado como não acessível. A partir da teoria desenvolvida por [Granollers, 2004] que utiliza o método de $\mathrm{DCU}^{11}$ no projeto de desenvolvimento de objetos de aprendizagem e que será implementado de acordo com as características desse(s) usuário(s). Esta metodologia prevê também contemplar características distintas de pessoas que apresentam alguma necessidade especial sendo também foco deste artigo.

A validação desse objeto de aprendizagem é constante e faz parte de todo o processo desde a análise até a implementação do objeto. Ainda fazendo parte dessa validação, outro teste esta sendo desenvolvido com um sujeito do ensino técnico cego que fará a validação dessa etapa atual do objeto, tendo previsto mais dois sujeitos do

11 Design Centrado no Usuário entende que o usuário torna-se o foco de atenção e a implementação e funcionalidade do sistema. 
ensino fundamental, cegos e com baixa visão para fazer os posteriores testes de validação.

O usuário faz parte do processo tanto da criação do protótipo como da avaliação do OA, com isso o restante das fases de construção e das atividades relacionadas serão melhor desenvolvidas atendendo a necessidade explicitado pelo usuário na criação inicial do protótipo.

\section{- Etapa 4: Validar a aplicação do objeto de aprendizagem acessível com alunos cegos, videntes e com baixa visão em uma sala de aula inclusiva}

Entendemos que de nada adianta a validação desse objeto de aprendizagem com um sujeito somente. Para entendermos o processo de inclusão e fazermos parte dessa construção devemos fazer a implementação e validação desse objeto com vários tipos de sujeitos para que possamos atender à diversidade. E para que isso aconteça, a intenção desse estudo é aplicar o objeto de aprendizagem acessível em uma sala de aula inclusiva, com sujeitos cegos, com baixa visão e videntes. Só assim teremos um objeto que atenda a todos, sem diferença em interfaces, funcionalidade e conteúdo.

\section{Desdobramentos e considerações finais}

De acordo com todos esses apontamentos mostrados nesse artigo, pensamos que a construção de objetos de aprendizagem que atendam à diversidade, precisa ser pensado desde sua construção prevendo também a criação de várias mídias, atendendo assim o maior de número de alunos e suas necessidades. Essa convergência de mídias facilitará assim, a adaptação de objetos de acordo com a necessidade apresentada em sala de aula. De acordo com as etapas de reestruturação desse objeto de aprendizagem, prevemos em continuidade do estudo a implementação da APIs de acessibilidade que permitirão além da identificação do OA pelos leitores de tela, também a utilização de outras tecnologias assistivas como ponteira para deficientes motores e teclas de atalho no teclado. Esse processo de implementação de API está sendo realizada, com previsão de validação com os sujeitos citados no artigo. Como etapa final dessa construção também está previsto a aplicação de metadados de acessibilidade ao modelo já criado e padronizado do objeto. Essa etapa será a última a ser desenvolvida, para depois de ser testado com sujeitos, ser incorporado a um repositório ou ambiente virtual de aprendizagem para utilização em sala de aula presencial ou virtual. Retomando o que foi dito acima, esse processo de desenvolvimento pode ser aplicado à reengenharia tanto de software como de objetos de aprendizagem, tendo a intenção com isso, que outros software preveja a acessibilidade desde sua construção.

\section{Referências}

Associação Catarinense para Integração do cego. ACIC: Leitores de Tela - Disponível em: < http://www.acic.org.br/acessibilidade_leitoresdetela.shtml > - Data de acesso: 24 Jul. 2009. 
Bartlett,K. Common myths aout web acessibility. 1999. Disponível em: http://aware.hwg.org/why/myths.html Acesso em 10 dez. 2008.

Behar, P.; Passerino, L.; Dias, C.; Frozi, A.P.; Silva, K.K. Um Estudo sobre requisitos pedagógicos para objetos de aprendizagem multi-plataforma. IFIP World Conference on Computer in Education 2009. Bento Gonçalves, jul 2009.

Chilkofsky, E.J; Cross, J.H. Reverse engineering and design recorvery: A taxonomy. IEEE Software, 7(1):13-18, janeiro de 1990.

Coleman, D. Desenvolvimento orientado a objetos: o método fusion. Rio de Janeiro: Editora Campus, 1996.

Conforto, D.; Santarosa, L. M. C. Acessibilidade à Web: Internet para Todos. Revista de Informática na Educação: Teoria, Prática - PGIE/UFRGS v. 5 nº2, p.87102. 2002.

Dias e Passerino. Objetos de Aprendizagem e Acessibilidade: um estudo sobre objetos acessíveis. XIX Simpósio Brasileiro em Informática na Educação. Fortaleza Nov 2008.

Dias, C. Usabilidade na Web: Criando portais acessíveis. Alta Books, 2003.

Korte, W. B., Hüsing, T. Benchmarking Access and Use of ICT in European Schools 2006: Results from Head Teacher and A Classroom Teacher Surveys in 27 European Countries, Ago 2006.

Gnome Live Java Access Bridge. Disponível em: http://live.gnome.org/Java\%20Access\%20Bridge. Acesso em 16 Jul 2009.

Granolllers, T. MPIu Uma metodologia que integra la ingenieria del software, la interacción persona-ordenador y la accesibilidad en el contexto de equipos de desarrollo multidisciplinares. Tesis de doctorado, Universidad de Lleida, julio 2004.

Java Accessibility Quick Tips - Ensuring and Verifying Basic Application Accessibility. Disponível em: http://www.sun.com/accessibility/docs/java access tips.jsp. Acesso em: 16 Jul. 2009.

Orca Leitor de Tela - Disponível em: http://live.gnome.org/Orca. Acesso em: 24 Jul. 2009.

RIVED - Rede Interativa Virtual de Educação. Disponível em: http://www.rived.mec.gov.br/ Acesso em: 12 nov. 2007.

Sonza, A. P. (2008) Ambientes Virtuais Acessíveis sob a perspectiva de Usuário com Limitação. Tese (Doutorado). Universidade Federal do Rio Grande do Sul, Programa de Pós-Graduação em Educação, Porto Alegre, 2008.

Sun - Accessibility - Disponível em: http://www.sun.com/accessibility - Acesso em: 16 Jul. 2009.

Sun- Developing Accessible JFC Applications - Test Cases. Disponível em: http://www.sun.com/accessibility/docs/dev_access_apps.jsp\#4 - Acesso em: $16 \mathrm{Jul}$. 2009.

Takahashi, T. Programação Orientada a Objetos, Escola de Computação, São Paulo, 1990. 
W3C/WAI. Web Accessibility Initiative Disponível em: http://www.w3.org/WAI Acesso em: 06 abr. 2008.

WARSCHAUER, Mark. Tecnologia e inclusão social. A exclusão digital em debate.

São Paulo: SENAC, 2006.

Willey, D. A. Connecting learning objects to instructional design theory: A definition, a metaphor, and taxonomy (2002). Disponível em: http://reusability.org/read/chpters/wiley.doc. Acesso em 05 nov. 2007.

ZABALLA, Antoni. A prática educativa: como ensinar. Art med. Porto Alegre,1998. 